\title{
Integración de las tecnologías de la información y la comunicación para la enseñanza de la lengua
}

\author{
Integration of Information and Communication \\ Technologies for Language Teaching
}

Yudi Herrera*

Resumen

En el presente artículo se realiza una revisión sobre la integración de las TIC para la enseñanza de la lengua. Este texto se estructura en tres secciones en las cuales se promueve el uso educativo de las nuevas tecnologías como herramientas cognitivas, es decir, cuya funcionalidad sea ampliar, potenciar y reorganizar las capacidades de los estudiantes no como simple contenedor o transmisor de información.

Además se presenta una propuesta de análisis del software educativo desde el enfoque pedagógico, privilegiando a aquellos que promuevan el aprendizaje reflexivo. Finalmente, se describen tres propuestas para integrar las TIC para la enseñanza de las lenguas por medio de simuladores, WebQuest y Wikis.

\section{Palabras claves:}

Integración curricular de TIC, las TIC como herramientas cognitivas, enseñanza de las lenguas con TIC, enseñanza de lenguas.

\section{Abstract}

This article presents a review of the integration of ICT in language teaching. This text is structured into three sections which promote the educational use of new technologies as cognitive tools, in other words expand the functions of cognitive tools, enhance and reorganize the capabilities of students not as a mere container or transmitter of information. In addition, this article presents an analysis of educational software from the pedagogical approach, focusing on those that promote reflexive learning. Finally, three proposals are discussed for the integrating of ICT for teaching languages through simulators, WebQuest and Wikis.

\section{Keywords:}

Cognitive tools, integration of ICT in education, integration of ICT in language learning and teaching, teaching of language.

Artículo recibido el 24 de mayo de 2007 y aprobado el 31 de octubre de 2007

* Magíster en Informática Educativa de la Universidad Tecnológica Metropolitana, Santiago de Chile. yherrera@udelmar.cl 


\section{Introducción}

La reflexión respecto a la manera adecuada de usar las Tecnologías de la Información y la Comunicación (TIC) en educación, nos ha traído por un recorrido que va desde la simple vitrina de sembrar computadores en laboratorios y en salas, hasta el debate respecto a la pertinencia de abrir cursos destinados a enseñar computación, o integrarlos como recurso didáctico en cada asignatura. Hemos recorrido un desorientado camino de dictados en computador, dibujos a trazo de teclado, lectura de cuentos largos y extenuantes ante la pantalla y a pesar de ello, aún nos preguntamos si para eso sirve la tecnología en educación.

En verdad, no hay claridad total sobre cuál sea esa mágica fórmula de integración; pero en los últimos años se observa una tendencia respecto a la finalidad de esta inserción. La idea que prevalece es que las destrezas informáticas acompañen al estudiante en la construcción de aprendizajes. Es decir, no restringir los computadores a clases que tratan sobre qué son y cómo se usan; sino hacer uso de las potencialidades de estas herramientas para generar aprendizajes e integrarlos en los momentos en los que se considera enriquecedores y pertinentes en las distintas áreas del currículo.

Cuando hablamos de integración curricular nos referimos a la relevancia de integrar las TIC y embeberlas en el desarrollo curricular. El propósito es la actividad de aprendizaje, la acción pedagógica, el aprender, y las TIC acuden como herramientas que vehiculan aquello. Las TIC se utilizan para fines curriculares, para apoyar una disciplina o un contenido curricular. Son herramientas para estimular el desarrollo de aprendizajes de alto orden. Cuando existe integración curricular de TIC estas se tornan invisibles, el profesor y el aprendiz se apropian de ellas y las utilizan en un marco situado del aprender (Sánchez, 2003, p. 50).

En el contexto de la enseñanza de la lengua, las TIC pueden llegar a ser la solución a distintos problemas educativos, si se planifica su entrada al currículo con propósitos como posibilitar la creación, la comunicación, la estructuración y reescritura de textos. El enfoque ( $\tan$ difundido) de incluir los computadores para luego buscar los aprendizajes a enseñar, ha demostrado la subutilización de estos recursos, la cual es producto de confundir los medios con los fines. Es que para usar computadores en educación no basta con mantenerlos encendidos en clase.

Antes de conectar los cables y poner a funcionar los procesadores, una institución que quiere abordar la integración de TIC debe lograr un consenso con su comunidad educativa respecto a cómo se entenderá dicha integración. A continuación se describen algunos factores a contemplar para integrar TIC al currículo.

Figura 1. Factores a contemplar para la integración curricular de TIC.

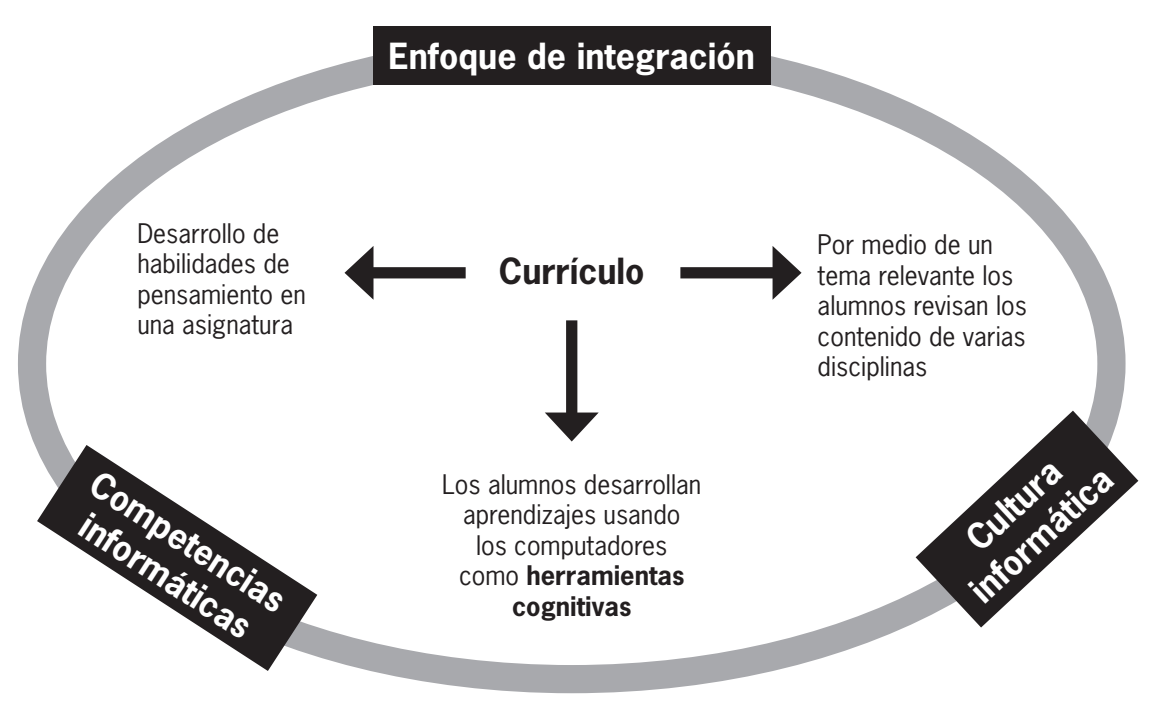


- Enfoque de integración de las TIC: es deseable que la comunidad involucrada valore las posibilidades didácticas de las TIC en el proceso educativo y teniendo en cuenta los objetivos de la institución. Deberá discutirse y llegar a un consenso respecto de las TIC y el currículo: si el currículo orienta el uso de las TIC o viceversa. Un acuerdo que implica dirigir los proyectos desde una concepción centrada en las TIC a una concepción centrada en el aprender con instrumentos tecnológicos.

- El desarrollo de las competencias tecnológicas y cultura informática en docentes y estudiantes de la institución. Se debe generar hábitos administrativos informáticos hacia los alumnos y docentes para incentivar la cultura informática, a través del envío de información por correo electrónico, la inscripción de asignaturas y la toma de algunos electivos a distancia. También, es adecuado mantener un acompañamiento permanente (un facilitador a disposición de los docentes) para la selección, adaptación y creación de recursos informáticos para su integración en el currículo.

- Diseño de integración curricular TIC: decidir qué tipo de integración curricular de las TIC se puede efectuar (Sánchez, 2003): dentro de una misma asignatura, para aprender contenidos o desarrollar habilidades, o tal vez para integrar a través de un tema varias asignaturas en las que las TIC serán sólo un medio

- Diseño de actividades curriculares en relación con el desarrollo de aprendizajes con uso de TIC: el papel de las tecnologías en el aprendizaje no es intentar la instrucción de los estudiantes, más bien, consiste en servir de herramientas de construcción del conocimiento, para que los estudiantes aprendan con ellas.

\section{El uso de las nuevas tecnologías en la generación de aprendizajes: las TIC como herramientas cognitivas}

Integrar computadores en educación no es un tema que se limite a su presencia física sino que exige ante todo claridad respecto a lo que es la esencia del proceso educativo que cada organización desea propiciar, de sus fines últimos, y entendimiento de las oportunidades que brinda la tecnología informática y de los requerimientos para hacerlas efectivas.

La misión central de la educación, según Brunner (2000), es la preparar para la vida, desarrollando procesos de pensamiento crítico e innovador, nutriendo la capacidad de escuchar y de comunicarse con personas que pueden tener puntos de vista contrapuestos sobre el mundo y sobre el bien, desarrollar la capacidad de aprender a aprender cuando se es confrontado con lo novedoso y con la necesidad de adaptación personal y social; ello en oposición a pensar la educación como la adquisición de un conjunto de ideas y hábitos derivados de la tradición y que, quienes tienen autoridad, asumen como válidos y deseables.

El poder intrínseco que tiene la tecnología informática es hacer posible una gran variedad de aproximaciones al problema educativo. Los enfoques algorítmico y heurístico se complementan, aunque se contraponen, y es posible hacer uso selectivo de ellos, dependiendo de que se trate de transmitir la herencia cultural o de desarrollar nuevos modelos de pensamiento, de liberar el potencial humano. Con apoyo de tecnologías de la información es posible crear ambientes educativos en que equipos, programas y personas -educadores y educandosinteractúen.

Por otra parte, el beneficio de las tecnologías no necesariamente debe remitirse a mediar cada una de las experiencias educativas que se diseñan en las instituciones. Muchas de ellas son susceptibles de llevarse a la práctica con apoyo de otros medios, algunos interactivos, como las experiencias directas con el objeto de conocimiento, otros transmisivos, como pueden ser los textos, los audiovisuales, las demostraciones y las explicaciones que provienen de diversas fuentes. El profesor es quien dispone las condiciones para que el alumno viva las experiencias deseables y necesarias para su aprendizaje. De allí que las múltiples ventajas de los medios informáticos, sólo lo serán en la medida en que para aprender un contenido específico, no exista mejor alternativa para propiciar el logro de los objetivos propuestos. 
Algunas de las ventajas de las tecnologías en educación han sido resumidas según Sánchez (1993) en:

- La interacción entre alumno y computador, que potencian un papel más activo de éste.

- Aumenta (si existe la cantidad de computadores necesaria) las posibilidades de tener una atención más personalizada.

- Existen posibilidades de realizar experiencias en ambientes controlados (sobre todo con los simuladores y los micromundos).

- El computador puede convertirse en una herramienta intelectual al estimular el desarrollo de estructuras lógicas (por ejemplo con el uso del software Logo) y al permitir mayor dinamismo en el procesamiento de información.

- Control del tiempo, contenido y de la secuencia de aprendizajes (en el software de tipo tutorial).

- Uso de la evaluación como medio de aprendizaje.

La postura frente al uso de tecnologías y su integración al currículo en este artículo comparte las bases teóricas propuestas por Jonassen y Reeves (1996) y Dede (2000) las cuales abogan por entender su papel no como transmisores de información sino como desarrolladores de habilidades de orden superior.

Jonassen (2000) afirma que toda actividad tiene una demanda cognitiva específica, para la cual los alumnos deben poder ejecutar las competencias adecuadas en un grado básico o avanzado. Las herramientas cognitivas tienen como propósito abordar y facilitar tipos específicos de procedimientos cognitivos; es decir, son dispositivos intelectuales que permiten representar, organizar, reestructurar, evaluar, calcular o realizar alguna acción que ayude a la labor cognitiva. Las herramientas cognitivas permiten visualizar más claramente una situación problemática, pueden servir para fortalecer o corregir esquemas cognitivos previos, pueden contribuir a comprender e interpretar información para resolver un problema.

La premisa que sustenta el uso de las TIC en educación desde el punto de vista de Jonassen (1994) es otorgarle a las tecnologías el papel de herramientas cognoscitivas más que de medios instruccionales. Habitualmente, los diseñadores de software preveían la presentación de información sobre diversos temas en contenedores de multimedia, la propuesta de Jonassen consiste en darles las herramientas a los aprendices para que ellos diseñen la representación de sus aprendizajes. Los estudiantes usarán la tecnología para analizar el mundo, obtener información, interpretar y organizar el conocimiento que van adquiriendo y mostrar a otros sus aprendizajes.

Jonassen (1996) señala que los computadores como herramientas cognitivas sirven fundamentalmente para ampliar, potenciar y reorganizar las capacidades de los estudiantes no como simple contenedor o transmisor de información. También postula que el papel de la tecnología desde este punto de vista permite desarrollar las habilidades de orden superior específicas que apoyan el desarrollo del pensamiento crítico entendiendo como tal “(análisis, evaluación y conexión) el pensamiento creativo (elaborar, sintetizar e imaginar) y el pensamiento complejo (diseñar, resolver problemas y tomar decisiones)" (Beltrán, 2004, p. 5).

Las herramientas cognitivas guían los procesos de pensamiento del alumno mientras aprende, realizando tareas de apoyo sin hacer que el estudiante dependa totalmente de la herramienta, es decir, que se sienta incapaz de realizar esta tarea sin ella, y menos eliminar la responsabilidad del alumno sobre su propio aprendizaje. Una de las críticas sobre los enfoques constructivistas es que al favorecer la actividad de los estudiantes a veces incurren en un activismo desmedido, sin sentido. En ocasiones, los estudiantes al finalizar la lección reconocen la realización de actividades parciales y no logran explicar la finalidad, su funcionalidad ni su pertinencia curricular.

Beltrán (2003), citando a Salomón, contrapone las actividades propias del pensamiento humano ante las actividades propias de los computadores, que en muchos paradigmas se confunden y se dejan, estas últimas, como metas para los alumnos.

Es más propio del alumno planificar, organizar, decidir, evaluar (tareas que caen bajo la responsabilidad del ser humano y que este sabe hacer mejor); 
y es más propio del ordenador almacenar, recuperar (tareas en las que el ordenador no tiene rival). Es más propio del ordenador realizar tareas de memoria, $y$ más propio del estudiante asumir tareas que exigen pensar y tomar decisiones (Beltrán, 2003, p. 2).

Con estos argumentos se fundamenta la necesidad de asignar y aprovechar las habilidades del alumno y del computador en tareas inteligentes $y$ pertinentes.

Con el software educativo quienes más aprenden son los diseñadores, puesto que el proceso de articular su conocimiento para representarlo en dicho software de instrucción, les hace desarrollar en ellos mismos un proceso de aprendizaje de mayor envergadura que el que emplean los estudiantes al observar estas representaciones.

Los estudiantes aprenden cuando se involucran en experiencias significativas, cuando tratan de representar de diversas maneras lo que saben. Los estudiantes deben utilizar tecnologías como herramientas, así como los profesionales emplean algunas herramientas como metros, mapas o maquetas para dar soluciones; los estudiantes beben emplear la tecnología, no como profesores particulares o depósitos de información.

Las herramientas y los ambientes cognoscitivos activan estrategias de aprendizaje y de pensamiento crítico. En el proceso del desarrollo cognoscitivo para el aprendizaje, la información debe tratarse de manera más profunda, se deben activar los modelos mentales apropiados, usarlos para interpretar la nueva información, asimilar la nueva información dentro de esos modelos previos, reorganizar los modelos a la luz de la información nueva y después de usar esos modelos enriquecidos para explicar, para interpretar, o para deducir nuevo conocimiento. $\mathrm{La}$ adquisición y la integración de conocimiento, según estas definiciones, es un proceso constructivo, así que al usar herramientas cognoscitivas, los estudiantes optimizan la construcción del conocimiento más que la reproducción del conocimiento.

Lo que el estudiante aprende, de acuerdo con la teoría constructivista, depende de lo que el alumno ya sabe, del tipo de experiencias que haya tenido y de cómo organiza estos conocimientos en su mente. Las herramientas cognoscitivas son para ayudar a principiantes a organizar y a representar lo que saben. De acuerdo con el constructivismo es muy difícil aprender la realidad, lo que hacemos es una interpretación de nuestras percepciones acordes con nuestras creencias. Si el aprendizaje se desarrolla solamente a través de la intervención personal en el mundo y la acomodación cognitiva de las interpretaciones, entonces el aprendizaje no se puede transferir de unas estructuras mentales de los docentes a otras de los alumnos.

En este contexto teórico, la labor docente será la de crear oportunidades de aprendizaje en las cuales los estudiantes participen con la guía del docente. El principio activo de este tipo de instrucción reside no tanto en las múltiples actividades planificadas para la interacción del alumno, sino en su papel agentivo de éste para participar en un contexto, de realizar acciones que se traduzcan en actividad cognitiva para apropiarse de los aprendizajes.

Norman (1993) discute dos tipos de pensamiento: experimental y reflexivo. El pensamiento experimental está relacionado con la experiencia del estudiante en el mundo, este tipo de pensamiento guía la conducta, emerge y a veces aparece de manera automática; mientras que el pensamiento reflexivo requiere del análisis, de la realización de procesos inferenciales y de la elaboración de estructuras conceptuales relacionales que se construyen y desestructuran permanentemente. El pensamiento reflexivo es una forma cuidadosa de pensamiento, requiere de la deliberación y generalmente de apoyo externo, por ejemplo de libros, de los computadores, de expertos $\mathrm{u}$ otras personas. Los computadores apoyan el pensamiento reflexivo, cuando permiten a las personas componer nuevos conocimientos, haciendo nuevas representaciones, modificando los escritos anteriores, en la reescritura, por ejemplo.

La perspectiva desde la cual se aborda la tecnología evidencia tres maneras de desarrollar el pensamiento de los alumnos: 
Cuadro 1. Perspectivas de abordaje pedagógico de los computadores en educación.

\begin{tabular}{|c|c|c|}
\hline Perspectiva & Objetivo & Descripción \\
\hline $\begin{array}{l}\text { Aprender sobre la } \\
\text { tecnología }\end{array}$ & Alfabetización informática & $\begin{array}{l}\text { Los estudiantes aprenden a desentrañar los componentes del hardware y a } \\
\text { programar. }\end{array}$ \\
\hline $\begin{array}{l}\text { Aprender de la } \\
\text { tecnología }\end{array}$ & Aprendizaje asistido por ordenador & $\begin{array}{l}\text { En este modelo el estudiante aprende del computador bajo el paradigma } \\
\text { de transmisión de conocimiento. En este tipo se encuentra el software de } \\
\text { ejercitación, la tutoría y los tutores inteligentes. El software de ejercitación } \\
\text { se presentaba a los estudiantes determinados problemas para ser resueltos, } \\
\text { introduciendo sus respuestas y el computador corregía las respuestas e } \\
\text { introducía el refuerzo correspondiente. Los tutoriales adaptaban su enseñanza } \\
\text { a las distintas capacidades, ritmos, estilos e intereses de cada estudiante. El } \\
\text { computador informaba, preguntaba y evaluaba las respuestas del alumno para } \\
\text { permitir avanzar a un nuevo nivel al alumno o no. Algunas de las críticas a estas } \\
\text { perspectivas de uso del computador son que dejan las decisiones educativas en } \\
\text { manos de máquinas sin inteligencia, y despojan de la capacidad de decisión a } \\
\text { alumnos inteligentes. }\end{array}$ \\
\hline $\begin{array}{l}\text { Aprender de la } \\
\text { tecnología }\end{array}$ & $\begin{array}{l}\text { El computador como herramienta } \\
\text { cognitiva }\end{array}$ & $\begin{array}{l}\text { El computador desarrolla el pensamiento del alumno en un nivel de aprendizaje } \\
\text { en el que no se considera un logro sólo la productividad del usuario, sino } \\
\text { la calidad del pensamiento. Es decir, cuando los estudiantes trabajan con } \\
\text { informática, en vez de ser controlados por ella, realzan las capacidades del } \\
\text { computador, y el computador realza su pensamiento y aprender mediante la } \\
\text { promoción de estrategias de reflexión, discusión y resolución de problemas. } \\
\text { Mientras que los estudiantes deben asignárseles la responsabilidad de } \\
\text { reconocer y de juzgar patrones de la información y después de organizarlos; al } \\
\text { computador le competen las tareas de memorización (pues tienen una enorme } \\
\text { capacidad para ello), realizar cálculos, almacenar y recuperar la información. } \\
\text { Cuando las herramientas cognoscitivas funcionan como socios intelectuales, } \\
\text { los logros de los estudiantes se realzan. Sin embargo, no deben confundirse } \\
\text { los conocimientos básicos con los cognitivos, los dos son necesarios. Los } \\
\text { conocimientos cognitivos son andamios para fundar la creación y de la reflexión } \\
\text { para generar nuevos conocimientos. }\end{array}$ \\
\hline
\end{tabular}

Fuente: Beltrán (2003).

Los efectos positivos del uso de TIC no sólo se restringen a los ámbitos del logro de aprendizajes, aumento de la motivación de los estudiantes, impactos en la metodología docente, sino que se ha comprobado cierto tipo de efectos cognitivos, es decir, se pueden encontrar efectos positivos sobre el conocimiento adquirido, el acceso al conocimiento y sobre la organización de los esquemas de conocimiento.

Perkins (1986) señala que "las escuelas pueden ayudar a los estudiantes a ser mejores pensadores añadiendo deliberadamente tácticas y estrategias inventadas por el hombre para organizar su pensamiento" (Perkins, 1986, p. 4). El efecto de las TIC en los marcos de pensamiento se refiere a la optimización de los procesos de organización y los procedimientos de cognición.

Entre los efectos positivos del uso de tecnología, mencionados por Perkins, están:
- La creación de metáforas que le sirven a los estudiantes para analizar, comprender e interpretar los fenómenos.

- La estimulación de diferenciaciones que generan nuevas categorías cognitivas.

- Potencian la actividad intelectual.

- Potencian habilidades específicas y activación de estrategias como las metacognitivas

- La internación de herramientas simbólicas que sirven de herramientas cognitivas.

Existen también otro tipo de efectos cognitivos que ocurren por el contacto con la tecnología como el favorecimiento de las habilidades comunicativas: rapidez, claridad y eficacia; además de otros efectos cognitivos más profundos: el fortalecimiento de la generación de puntos de vista, estrategias de pensamiento lógico, estrategias metacognitivas, de resolución de problemas, etc. 
Dentro de las actividades de aula que los docentes desarrollan existe un amplio espectro, nuestro interés es favorecer todas las actividades que privilegian el desarrollo de habilidades cognitivas (denominadas de desafío cognitivo) (cuadro 2).

Cuadro 2. Práctica docente de aula: clasificación de estrategias de aprendizaje de la práctica docente.

\begin{tabular}{|c|c|c|c|c|c|}
\hline \multicolumn{3}{|c|}{ Desafío cognitivo } & \multirow{2}{*}{\multicolumn{2}{|c|}{$\begin{array}{l}\text { Estrategias de aprendizaje } \\
\text { Activo }\end{array}$}} & \multirow{3}{*}{$\begin{array}{c}\text { Enfoque } \\
\text { tradicional } \\
\begin{array}{c}\text { Actividad } \\
\text { tradicional }\end{array}\end{array}$} \\
\hline \multicolumn{2}{|c|}{ Estrategias de aprendizaje reflexivo } & \multirow{2}{*}{$\begin{array}{c}\begin{array}{c}\text { Estrategias de } \\
\text { transición }\end{array} \\
\begin{array}{c}\text { Resolución de } \\
\text { problemas }\end{array} \\
\end{array}$} & & & \\
\hline $\begin{array}{l}\text { Actividades para } \\
\text { el desarrollo del } \\
\text { pensamiento }\end{array}$ & $\begin{array}{l}\text { Escritura } \\
\text { reflexiva }\end{array}$ & & Proyectos & Trabajo grupal & \\
\hline $\begin{array}{l}\text { Los estudiantes justifican y } \\
\text { explican su razonamiento } 0 \\
\text { realizan debates. }\end{array}$ & $\begin{array}{l}\text { Los estudiantes } \\
\text { escriben un } \\
\text { diario o un } \\
\text { ensayo. }\end{array}$ & $\begin{array}{l}\text { La frecuencia } \\
\text { con la que los } \\
\text { estudiantes trabajan } \\
\text { en la resolución de } \\
\text { problemas, los cuales } \\
\text { no tienen un método } \\
\text { obvio o solución. }\end{array}$ & $\begin{array}{l}\text { Trabajos } \\
\text { prácticos o en } \\
\text { laboratorios }\end{array}$ & $\begin{array}{l}\text { Los estudiantes } \\
\text { trabajan en } \\
\text { pequeños grupos } \\
\text { completando una } \\
\text { tarea como equipo. }\end{array}$ & $\begin{array}{l}\text { El docente dirige una } \\
\text { discusión frente a la } \\
\text { clase completa(los } \\
\text { estudiantes escuchan } \\
\text { y responden las } \\
\text { preguntas). }\end{array}$ \\
\hline $\begin{array}{l}\text { Las razones por las que los } \\
\text { docentes realizan preguntas } \\
\text { a sus estudiantes es } \\
\text { para... conocer sus ideas y } \\
\text { opiniones. }\end{array}$ & $\begin{array}{l}\text { Los estudiantes } \\
\text { explican su } \\
\text { pensamiento en } \\
\text { amplitud. }\end{array}$ & $\begin{array}{l}\text { Haber diseñado sus } \\
\text { propios problemas por } \\
\text { resolver. }\end{array}$ & $\begin{array}{l}\text { Trabajo en } \\
\text { proyectos de una } \\
\text { semana o más. }\end{array}$ & $\begin{array}{l}\text { Los estudiantes } \\
\text { trabajan en grupo } \\
\text { para solucionar un } \\
\text { problema o cumplir } \\
\text { una tarea. }\end{array}$ & $\begin{array}{l}\text { Los estudiantes } \\
\text { responden } \\
\text { individualmente las } \\
\text { preguntas de una guía } \\
\text { o texto. }\end{array}$ \\
\hline $\begin{array}{l}\text { Los estudiantes han } \\
\text { relacionado sus aprendizajes } \\
\text { con su propia experiencia. }\end{array}$ & $\begin{array}{l}\text { Los estudiantes } \\
\text { escriben un } \\
\text { ensayo o una } \\
\text { evaluación } \\
\text { respecto a su } \\
\text { propio trabajo. }\end{array}$ & $\begin{array}{l}\text { Decidir los } \\
\text { procedimientos para } \\
\text { resolver problemas } \\
\text { complejos y discutir } \\
\text { sus resultados. }\end{array}$ & $\begin{array}{l}\text { Hacer un } \\
\text { producto para } \\
\text { ser utilizado por } \\
\text { alguien más. }\end{array}$ & & \\
\hline $\begin{array}{l}\text { Los estudiantes sugieren o } \\
\text { colaboran en la planificación } \\
\text { de las temas o actividades de } \\
\text { clases. }\end{array}$ & & & $\begin{array}{l}\text { Presentar su } \\
\text { trabajo ante } \\
\text { una audiencia } \\
\text { incluyendo } \\
\text { personas } \\
\text { externas de la } \\
\text { clase. }\end{array}$ & & \\
\hline \multicolumn{6}{|l|}{$\begin{array}{l}\text { Debatir y argumentar un } \\
\text { punto de vista, incluso uno no } \\
\text { compartido por él mismo. }\end{array}$} \\
\hline $\begin{array}{l}\text { Representar la misma idea } \\
\text { o relación en más de una } \\
\text { manera. }\end{array}$ & & & & & \\
\hline
\end{tabular}

Fuente: Becker (2003).

\section{Tipos de software}

\section{y su funcionalidad educativa}

Existen varias formas de analizar las maneras como los computadores se incorporan a la educación entre ellas la propuesta de Crook (1998) en la cual se puede relacionar el uso del computador, las concepciones pedagógicas y las prácticas de los docentes en el aula. Esta propuesta considera que el uso del software educativo se puede clasificar de acuerdo con tres papeles diferentes: actuar como un instructor de los alumnos, ser una herramienta de trabajo o actuar como un aprendiz de los estudiantes.

\section{El computador como instructor}

Es una metáfora en la cual el computador, siguiendo el enfoque tradicional, es el centro de la actividad lectiva, provee el conocimiento, posibilidades de aprendizaje y estrategias de evaluación. Basándose en una estructura prediseñada que no puede personalizarse ante cada uno de los estudiantes, se dedica 
a enseñar conceptos, a estimular la práctica de habilidades (ejercitación) y a evaluar el aprendizaje.

Esta modalidad es útil para el aprendizaje de destrezas específicas, pero deben ser empleados dentro de un diseño curricular. El aprendizaje de algunas destrezas específicas requiere de mucha práctica, de allí su popularidad; pero el computador puede repetir como producto cerrado las mismas preguntas, ayudas y niveles de ejercitación que agotan su potencial educativo.

\section{El computador como herramienta}

El computador puede proveer al estudiante de herramientas que faciliten las operaciones mecánicas, liberando su atención hacia actividades de un orden superior de pensamiento. Esta modalidad potencia el desarrollo de las habilidades del estudiante, ya que facilita su expresión y le permite revisar y corregir sus trabajos para mejorarlos.

El uso, por ejemplo, del procesador de palabras concentra los esfuerzos de los estudiantes en estructurar, planificar, producir y revisar el texto, corregirlo, eliminando la interferencia y las limitaciones impuestas por el acto motor de escribir. Esto mismo sucede cuando se emplean herramientas dentro de las asignaturas de cálculo y matemáticas, posibilitando la toma de decisiones, la interpretación de los datos más que el cálculo de los mismos.

Lograr una comunicación efectiva mediante el uso del lenguaje escrito suele ser una tarea desconocida para los estudiantes dentro del ambiente escolar, donde tradicionalmente la escritura tiene como único objeto obtener la calificación del maestro. Las redes de computadoras pueden ser utilizadas como herramientas de comunicación para intercambiar información, conectarse con otras personas y practicar las lenguas extranjeras en contextos reales, además, los estudiantes también pueden publicar sus trabajos en Internet contribuyendo también a generar y colaborar con su conocimiento.

\section{El computador como aprendiz}

Esta metáfora consiste en que el "computador es enseñado" por el estudiante. El alumno puede aplicar y probar sus ideas en mundos virtuales que siguen la dinámica de los sistemas reales, como en el caso de los simuladores, en donde el computador sigue las orientaciones de los estudiantes, y con las reglas propias del sistema va experimentando variaciones. Los simuladores son programas que han sido diseñados con la intención de «simular» o crear ficticiamente procesos reales con los que los estudiantes interactúan aprendiendo de los mundos simulados y de la interacción con ellos. Generalmente, este tipo de software es empleado en las áreas científicas en las que es difícil realizar experimentos reales para aprender conceptos complejos con los estudiantes, para aprender leyes físicas o de relaciones etológicas entre grupos de animales. Los estudiantes se introducen en estos mundos virtuales, interactúan con organismos, tratan de solucionar problemas e hipotetizan posibles causas y efectos que, mediante la experimentación simulada, comprueban finalmente.

Otro tipo de software aprendiz son los editores o programadores como el conocido LOGO, en los cuales los estudiantes "enseñan" a los computadores a realizar las acciones. Los alumnos para poder programar alguna acción se ven obligados a comprender los procesos implícitos de la acción y a hacerlos explícitos mediante la lógica de los computadores. Los estudiantes deben entonces razonar cuál es la secuencia de acciones que deben realizar para calcular un resultado y, además, comunicar las instrucciones a la computadora.

A continuación se presenta un cuadro que relaciona esta tipología de Crook, en la cual se explicitan las relaciones entre el uso del computador, las concepciones pedagógicas y las prácticas de los docentes en el aula (cuadro 3). Este cuadro nos permite explicitar los objetivos de aprendizaje y el enfoque pedagógico que subyace a la incorporación de las diversas aplicaciones informáticas. Se espera que los docentes privilegiemos las herramientas informáticas que estimulan el desarrollo del pensamiento, las que están ubicadas en la parte inferior del cuadro. 
Cuadro 3. Metáforas de uso del computador según paradigma educacional y metas de aprendizaje.

\begin{tabular}{|c|c|c|c|c|c|}
\hline $\begin{array}{l}\text { Papel del } \\
\text { computador }\end{array}$ & $\begin{array}{l}\text { Concepciones } \\
\text { pedagógicas }\end{array}$ & $\begin{array}{l}\text { Estrategias de práctica } \\
\text { docente }\end{array}$ & $\begin{array}{l}\text { Control de } \\
\text { situación de } \\
\text { Aprendizaje }\end{array}$ & $\begin{array}{c}\text { Metas del aprendizaje } \\
\text { para los estudiantes }\end{array}$ & Ejemplos \\
\hline $\begin{array}{l}\text { Tutor } \\
\text { (el computador } \\
\text { como máquina } \\
\text { de enseñanza) }\end{array}$ & $\begin{array}{l}\text { Enfoque } \\
\text { tradicional }\end{array}$ & $\begin{array}{l}\text { Estrategias tradicionales: } \\
\text { refuerza contenidos mediante } \\
\text { ensayo y error. }\end{array}$ & $\begin{array}{l}\text { El software } \\
\text { controla el ritmo y } \\
\text { el contenido. }\end{array}$ & $\begin{array}{l}\text { Aprendizaje de contenidos, } \\
\text { ejercitación de destrezas. } \\
\text { Autoaprendizaje. Objetivos } \\
\text { reducidos a los propósitos del } \\
\text { software. }\end{array}$ & $\begin{array}{l}\text { Software } \\
\text { tutorial. }\end{array}$ \\
\hline \multirow{2}{*}{$\begin{array}{l}\text { Aprendiz } \\
\text { (el alumno le } \\
\text { "enseña" a la } \\
\text { máquina, la } \\
\text { programa) }\end{array}$} & $\begin{array}{l}\text { Enfoque } \\
\text { constructivista } \\
\text { Aprendizaje por } \\
\text { descubrimiento }\end{array}$ & $\begin{array}{l}\text { Estrategias de aprendizaje } \\
\text { activo: } \\
\text { incorpora las fases del } \\
\text { método científico deductivo. } \\
\text { Actividades de resolución de } \\
\text { problemas. }\end{array}$ & $\begin{array}{l}\text { El alumno semi- } \\
\text { controla }\end{array}$ & $\begin{array}{l}\text { Aprendizaje de procedimientos } \\
\text { (más que de contenidos), } \\
\text { fenómenos, relaciones de } \\
\text { causa-efecto, deducción, } \\
\text { elaboración de hipótesis. } \\
\text { Objetivos reducidos a los } \\
\text { propósitos del software. }\end{array}$ & $\begin{array}{l}\text { Software de } \\
\text { simulación }\end{array}$ \\
\hline & $\begin{array}{l}\text { Enfoque } \\
\text { constructivista: } \\
\text { aprendizaje por } \\
\text { descubrimiento }\end{array}$ & $\begin{array}{l}\text { Estrategias de aprendizaje } \\
\text { Activo y de transición: } \\
\text { Elaboración de proyectos } \\
\text { mediante la estructuración } \\
\text { lógica y representación } \\
\text { simbólica de una secuencia } \\
\text { de acciones. }\end{array}$ & $\begin{array}{l}\text { El alumno semi- } \\
\text { controla }\end{array}$ & $\begin{array}{l}\text { Desarrollo de habilidades de } \\
\text { pensamiento formal más que } \\
\text { aprendizaje de contenidos } \\
\text { o procesos. Pensamiento } \\
\text { algorítmico, desarrollo } \\
\text { lógico y aprendizaje sobre la } \\
\text { tecnología. }\end{array}$ & $\begin{array}{l}\text { Software y } \\
\text { lenguajes de } \\
\text { programación }\end{array}$ \\
\hline $\begin{array}{l}\text { Caja de } \\
\text { herramientas } \\
\text { (máquinas } \\
\text { dedicadas) }\end{array}$ & $\begin{array}{l}\text { Enfoque } \\
\text { constructivista: } \\
\text { teorías de } \\
\text { desarrollo } \\
\text { cognitivo }\end{array}$ & $\begin{array}{l}\text { Estrategias de aprendizaje } \\
\text { reflexivo: trabajos en } \\
\text { proyectos y estrategias para } \\
\text { el desarrollo del pensamiento } \\
\text { uso, y análisis de información. }\end{array}$ & $\begin{array}{l}\text { El alumno } \\
\text { controla }\end{array}$ & $\begin{array}{l}\text { Desarrollo de las habilidades } \\
\text { superiores de pensamiento: } \\
\text { creatividad, pensamiento } \\
\text { crítico, toma de decisiones. } \\
\text { Amplio espectro de objetivos } \\
\text { de aprendizaje. }\end{array}$ & $\begin{array}{l}\text { Herramientas de } \\
\text { productividad y } \\
\text { editores. }\end{array}$ \\
\hline \multirow[t]{2}{*}{$\begin{array}{l}\text { Consulta } \\
\text { informativa } \\
\text { (instrumento } \\
\text { informativo) }\end{array}$} & $\begin{array}{l}\text { Enfoque } \\
\text { tradicional y } \\
\text { de aprendizaje } \\
\text { activo }\end{array}$ & $\begin{array}{l}\text { Estrategias de aprendizaje } \\
\text { activo } \\
\text { Estrategias de búsqueda, } \\
\text { selección. Trabajo en } \\
\text { proyectos. }\end{array}$ & $\begin{array}{l}\text { El alumno semi- } \\
\text { controla }\end{array}$ & $\begin{array}{l}\text { Promueve el desarrollo de } \\
\text { habilidades de autoaprendizaje }\end{array}$ & $\begin{array}{l}\text { Enciclopedias, } \\
\text { bases de datos. } \\
\text { Páginas Web, } \\
\text { e-learning. }\end{array}$ \\
\hline & & $\begin{array}{l}\text { Estrategias de aprendizaje } \\
\text { activo y reflexivo: }\end{array}$ & & & \\
\hline $\begin{array}{l}\text { Entorno de } \\
\text { comunicación } \\
\text { (medio de } \\
\text { comunicación) }\end{array}$ & $\begin{array}{l}\text { Enfoque } \\
\text { constructivista: } \\
\text { teorías socio } \\
\text { cognitivas. }\end{array}$ & $\begin{array}{l}\text { Trabajo en grupos } \\
\text { distribuidos distantes. } \\
\text { Distribución de conocimientos } \\
\text { colegiados. Énfasis en } \\
\text { el trabajo cooperativo y } \\
\text { colaborativo. }\end{array}$ & $\begin{array}{l}\text { El alumno } \\
\text { controla }\end{array}$ & $\begin{array}{l}\text { Construcción de conocimiento } \\
\text { con mediación social, } \\
\text { desarrolla habilidades sociales } \\
\text { y de trabajo colectivo. }\end{array}$ & $\begin{array}{l}\text { Comunidades } \\
\text { de aprendizaje, } \\
\text { weblogs, foros }\end{array}$ \\
\hline
\end{tabular}

\section{Aproximaciones del uso de tecnologías de la información y la comunicación para la enseñanza de las lenguas}

\section{El computador como aprendiz: integración de software de simulación en la enseñanza de las lenguas}

La capacidad del aprendiz para analizar todos los factores que confluyen en las situaciones y problemas es denominada flexibilidad cognitiva; la capacidad que tienen para utilizar y aplicar diversa información y generar conceptos complejos o poder aplicar este conocimiento a situaciones reales forman parte del propósito de usar las TIC como herramientas de la mente.

Una de las iniciativas más llamativas en la generación de herramientas para potenciar el pensamiento es la que desarrolla Intel ${ }^{1}$ de quienes se presentan a continuación tres propuestas:

1 Estas herramientas se pueden consultar en http://www.intel.com/ education/visualranking/index.htm 
Una herramienta de organización visual que permite al estudiante que ante una situación creada por el docente jerarquice factores y justifique sus propuestas, en este ejercicio el estudiante puede manipular sus ideas y comparar sus resultados con los de sus compañeros. Este ejercicio permite ejercitar la habilidades lógicas y la capacidad de investigar, analizar y decidir cómo jerarquizar una serie de factores de acuerdo a sus propios argumentos.

Figura 2. Herramienta de organización visual.

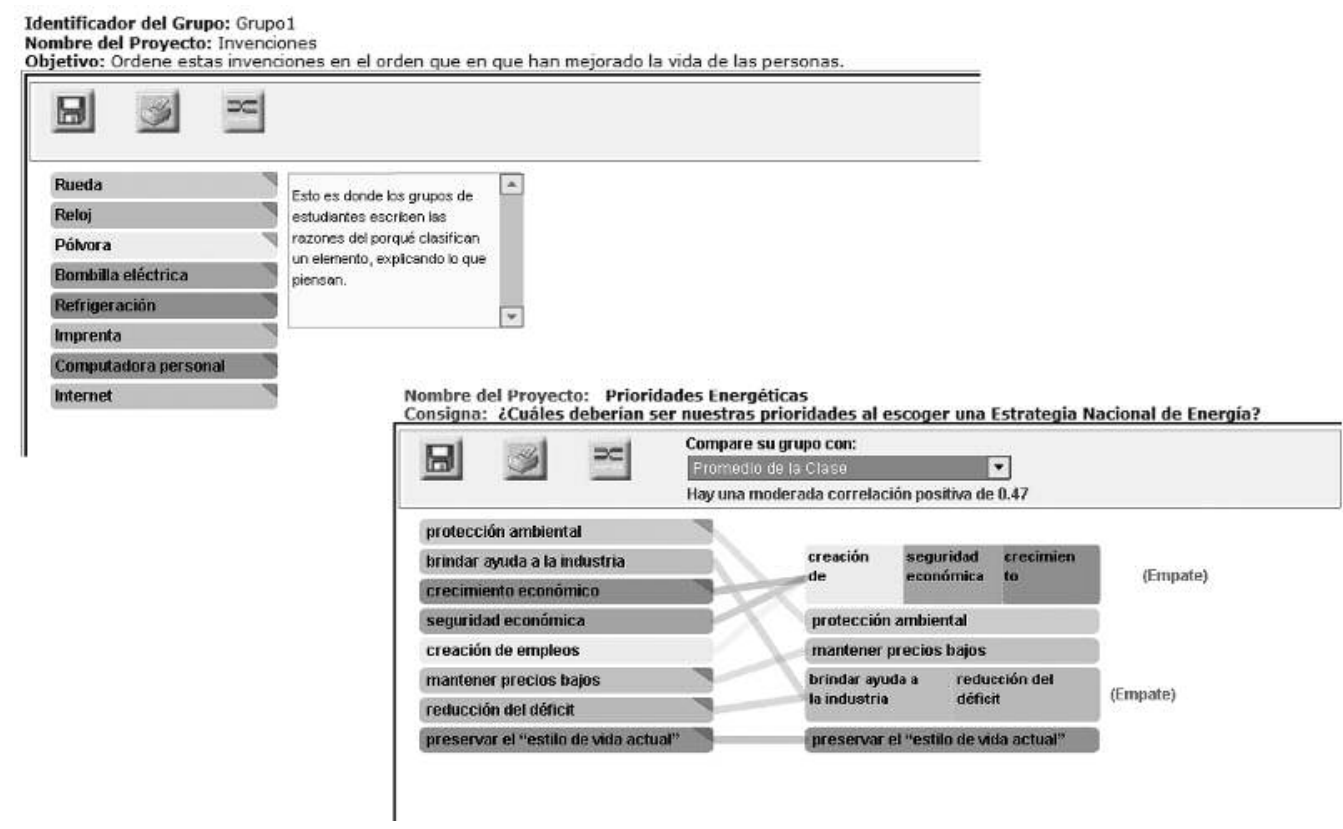

Fuente: Intel herramientas de pensamiento

Otra de las propuestas de Intel es la herramienta explicando una razón cuyo propósito es evidenciar las relaciones de causa y consecuencia entre factores, su interrelación y la manera como los estudiantes comprenden algunos fenómenos. La función del computador en este y el anterior caso es sólo servir como instrumento para organizar el razonamiento de los estudiantes.

Figura 3. Herramienta explicando una razón.

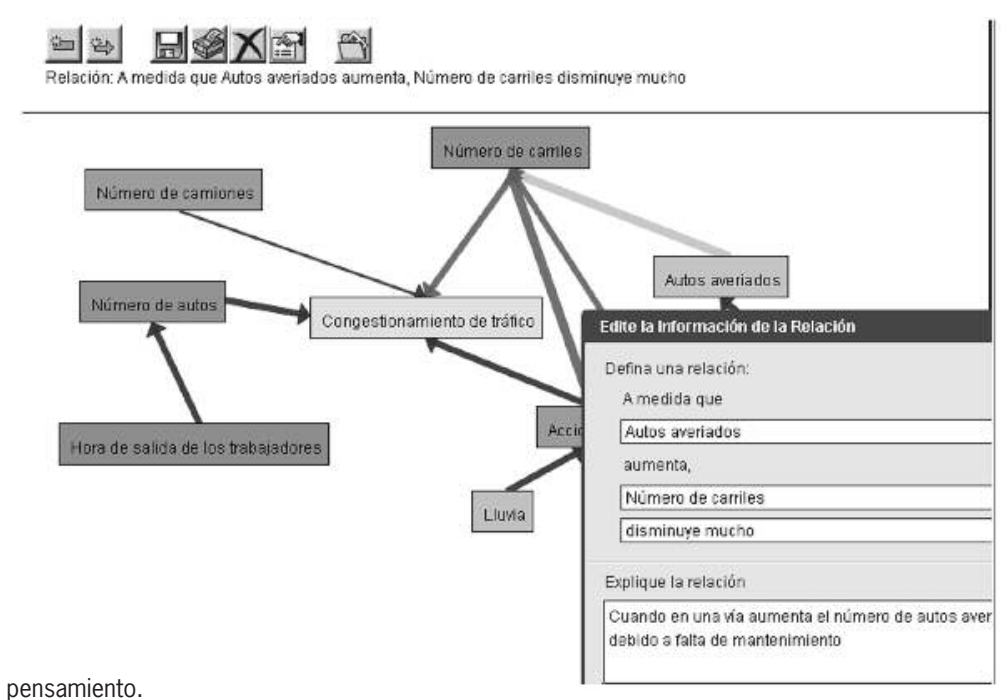

Fuente: Intel herramientas de pensamiento. 
Nuestros estudiantes encuentran notables dificultades para el desarrollo de las habilidades de comprensión y producción de discursos argumentativos, perceptibles incluso hasta el inicio de su actividad académica en la universidad. En el ejemplo anterior se espera que ante un problema, en este caso el congestionamiento del tráfico, los estudiantes sean capaces de identificar las causas, los efectos y establecer interrelaciones. En esta simulación no sólo se conectan y determinan los factores, sino que además se conceptualizan y se mide el grado de influencia entre unos y otros.

La tercera propuesta de Intel tiene por objeto desarrollar las habilidades argumentativas de los alumnos mediante la formulación de hipótesis y la proposición de argumentos.

A partir de la investigación y la proposición de un tema apropiado, los estudiantes deben generar hipótesis y construir evidencias con fundamento para probar sus argumentos y validar sus posturas. Estas posibilidades desarrollan las competencias comunicativas no sólo en la lengua nativa sino que permiten proyectar su uso en el aprendizaje de segundas lenguas. Como se muestra en la figura 4 los alumnos elaboran, documentan, organizan y califican la importancia de sus argumentos lo que posibilitaría la construcción de textos argumentativos.

Figura 4. Herramientas mostrando evidencias.

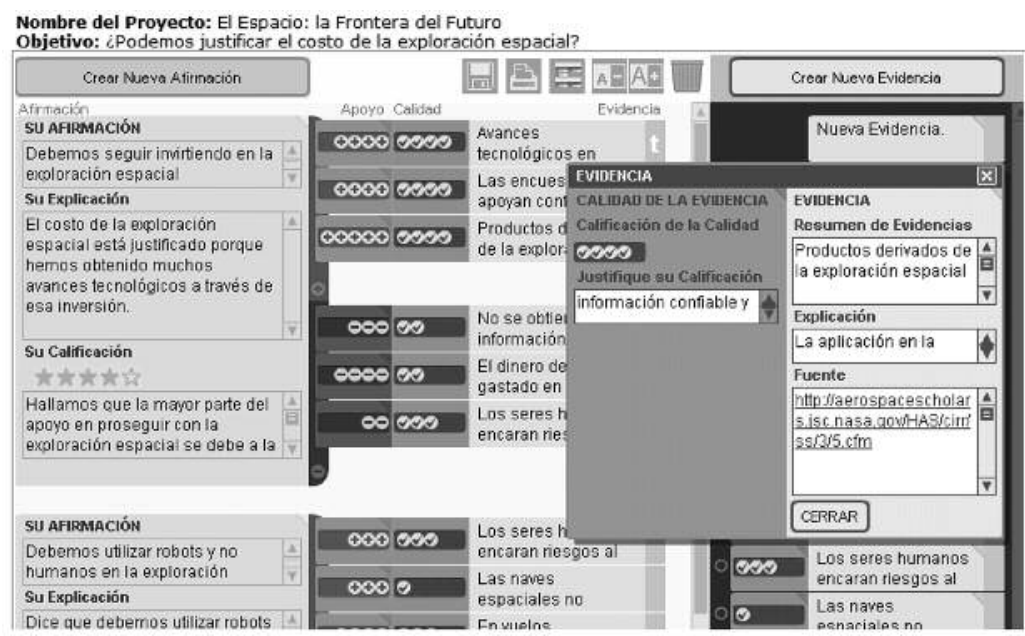

Fuente: Intel herramientas de pensamiento.

\section{Integración de WebQuest para guiar la gestión de información en la enseñanza de las lenguas}

La propuesta realizada por Dodge (2001), sobre estas guías hipermediales que organizan las búsquedas de los alumnos en el ciberespacio, les ayudan no sólo a tener más claridad sobre qué y dónde investigar, sino que garantizan a los estudiantes lograr un aprendizaje por descubrimiento con mayor eficiencia.

Un WebQuest es una guía de aprendizaje hipermedial propuesta como un desafío que los estudiantes deberán resolver a través de una navegación guiada por la web. Consta de cinco partes esenciales: introducción, tarea, procedimiento, recursos y evaluación. Cada una de ellas entrega una acotada orientación sobre cómo, dónde y para qué investigar.

\section{Nuevas maneras de escribir: aportes para un aprendizaje socio cognitivo}

El impacto en la manera de representar o de reorganizar el pensamiento, luego de la integración de una herramienta cognitiva, depende no sólo de las características de éstas sino del tipo de actividades que hagan uso de la misma, de la creatividad de los docentes. El trabajo colaborativo implica para los alumnos la negociación de metas, de los procedimientos de resolución y del análisis del problema. 
Los alumnos deben realizar un trabajo de comprensión común intercambiando argumentos y significados, en fin, una labor metacognitiva.

Las herramientas tecnológicas se constituyen en un ambiente simbólico de mediación que apoya la negociación de aprendizajes. En este escenario las publicaciones colaborativas y las herramientas de escritura colaborativa tienen una enorme transcendencia para el aprendizaje de la lengua. Incluso la actividad individual de la reescritura en un procesador de palabras, genera una revisión y autocrítica (actividad metacognitiva) para mejorar la escritura. La intervención continua y el proceso de creación sostenida en el tiempo de un texto, mejorándolo, es una evidencia constructivista de cómo se produce el aprendizaje.

Las herramientas Wiki, que son procesadores de texto compartidos, potencian los procesos de escri- tura colaborativa, es así como un grupo de personas puede crear, modificar e intervenir un mismo texto con aportes de cada individuo y cuya propiedad sobre las ideas puede identificarse gracias a las herramientas tecnológicas. Este tipo de herramientas cognitivas explicita los procesos de producción discursiva y visibiliza las negociaciones cognitivas de los estudiantes que intervienen en él.

Un ejemplo impactante de esta propuesta son las Wikinovelas (novelas escritas de manera colaborativa), las cuales han demostrado ser sumamente eficaces para hablar de los procesos colectivos de escritura. Miles de escritores leían y continuaban la temática de la novela generando espontáneos desenlaces para el entramado de la historia. Se dice que éstas son obras no tanto para ser leídas como para ser escritas por el tamaño del texto.

Figura 5. Wiki novela un espacio de escritura colaborativa.

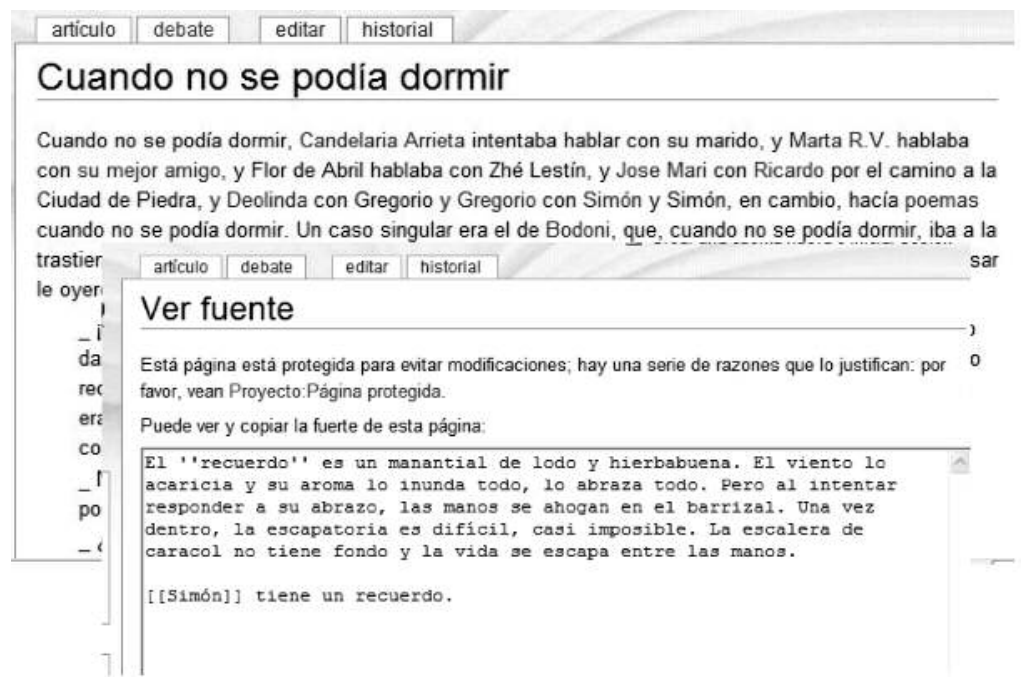

Fuente: WikinNovela Un millón de pingüinos².

Otras de las herramientas que permiten realizar propuestas de escritura colaborativa guíadas cronológicamente son los diarios de vida virtuales o Bloggers. Éstos son diarios hipermediales que permiten no sólo la escritura y publicación de textos de los estudiantes en el web; sino que posibilitan la

2 Esta wikinovela puede ser consultada en http://www.amillionpenguins.com/wiki/index.php/Main_Page socialización de reflexiones e investigaciones pedagógicas de los docentes. Dentro del uso en el aula se promueve la publicación y desarrollo de diarios escolares, diarios de vida, ensayos comentados, entre otras aplicaciones. Los foros de discusión también posibilitan un intercambio de opiniones más reflexivo y democratizan la participación de los estudiantes. 


\section{A modo de conclusión}

Ni los computadores ni Internet fueron creados originalmente con fines educativos; no obstante, su uso planificado ofrece amplias posibilidades, algunas de las cuales han sido integradas en este artículo.

Los efectos del uso de las tecnologías en la generación de aprendizajes no ocurren de manera espontánea, es necesario realizar una integración planificada y reflexiva, la cual integre una mirada a los enfoques pedagógicos y a los propósitos del aprendizaje curricular. Se ha presentado una propuesta que aboga por incorporar las tecnologías como instrumentos de desarrollo cognitivo, a través del desarrollo de actividades constructivas reflexivas.

Dentro de las herramientas que promueven un aprendizaje constructivista para el aprendizaje de las lenguas se destacan los ambientes que simulan y explicitan los razonamientos y argumentaciones de los estudiantes, para ayudarles a manipular las estructuras discursivas y a ejercitar su organización, herramientas desarrolladas por Intel.

De igual manera, se han enfatizado los ambientes que convocan y posibilitan la construcción colaborativa de hipertextos como una actividad sociocognitiva. Este artículo presenta una postura clara respecto al uso de las tecnologías de la comunicación y la información para el desarrollo de habilidades cognitivas y metacognitivas, y a la potenciación de la negociación y de la construcción de significados en las actividades colaborativas. Cuando las TIC apoyan el proceso de resolución colaborativa y de escritura generando representaciones manipulables de argumentos y posibilitando la negociación podemos sentir que integramos estos recursos de manera inteligente y significativa.

Aunque actualmente hay un menor uso de estrategias de aprendizaje reflexivo, que coincide con la menor proporción de uso pertinente de las TIC como herramientas cognitivas, la existencia de algunas de estas prácticas educativas muestra una transición hacia prácticas y concepciones más constructivistas y un uso de la tecnología más pertinente. Aún falta un amplio camino por desarrollar y diversificar en cuanto al tipo de usos posibles y pertinentes de las tecnologías para el aprendizaje de las lenguas en el contexto escolar y universitario. Se percibe un lento avance en el uso de recursos informáticos que transita desde los objetivos de transmisión de contenidos hasta el de desarrollo de habilidades de pensamiento, lo cual tiene una enorme relación con el tipo de concepciones, prácticas y conocimiento que desarrollan los docentes. El camino de mejora educativa a nivel pedagógico (desplazando los modelos tradicionalistas) será un indicador efectivo para señalar el mejor aprovechamiento y uso pedagógico de incorporación de las TIC en el currículo educativo. \}

\section{Bibliografía}

Becker, H. J. (1998). The influence of computer and Internet use on Teachers Pedagogical Practices and Perceptions. Paper presented at the annual meeting of the American Educational Research Association, San Diego, C.A.

Beltrán Llera, Jesús A. (2004). La familia ante los desafíos del siglo XXI. En: LXXXVIII Congreso Unión Nacional de Padres de Familia. Universidad Complutense de Madrid.

Beltrán Llera, Jesús A. (2003). Enseñar a aprender. Conferencia de Clausura del Segundo Congreso de Educared.
Brunner, J. J. (2000). Educación y escenarios futuros. Nuevas tecnologías y sociedad de la información. Seminario sobre Prospectiva de la Educación en la Región de América Latina y el Caribe. UNESCO: Santiago de Chile, 23 al 25 de agosto.

Brunner, J. J. (2003). Informe Capital Humano. Chile: Universidad Adolfo Ibáñez.

Crook, Charles. (1998). Ordenadores y aprendizaje colaborativo. Madrid: Ministerio de Educación y CulturaEdiciones Morata.

Dede, Chris. (2000). Aprendiendo con tecnología. Buenos Aires: Paidós. 
Dede, Chris. (2002). Emerging Influences of Information Technology on School Curriculum. Journal of Curriculum Studies, 32(2), 281-303.

Dodge, B. (2001). FOCUS: Five rules for writing a great WebQuest. Learning \& Leading with Technology, 28(8), 6-9. Consultado el 1 de septiembre en: http:// webquest.sdsu.edu/documents/focus.pdf.

Fulton, Katleen. (1999). How Teachers Beliefs about Teaching and Learning Are Reflected in their Use of Technology: Case Studies from Urban Middle Schools. Unpublished Master of Arts. College Park: University of Maryland.

Fullan, M. (2001). The New Meaning of Educational Change. Nueva York: Teachers College Press, Columbia University.

Hinostroza, J. E. (2001). Chile en Estudio Internacional Sites. Para usar mejor la tecnología en el aula. Revista del Ministerio de Educación de Chile. Consultado en diciembre de 2003 en: http://www.mineduc.cl/ revista/anteriores/agosto01/ tema2.htm].

Jonassen, David H. (1994). Technology as Cognitive Tools: Learners as Designers Pennsylvania State University ITForum Paper 1. Consultado en: http://itech1.coe. uga.edu/itforum/paper1/paper1.html.

Jonassen, David H. (2000). El diseño de entornos constructivistas de aprendizaje. En: Diseño de la instrucción. Teorías y modelos: un nuevo paradigma de la teoría de la instrucción, Charles Reigeluth (coord.). 1, 225-249. Madrid: Aula XXI Santillana.

Jonassen, David H. y Reeves, T. C. (1996). Learning with technology: Using computers as cognitive tools. En: D.H. Jonassen (ed.). Handbook of Research for Educational Communications and Technology, pp. 693-719. Nueva York: Macmillan.

Marion, Vosahlo. (2001). Mindtools.Technology Tools for Teaching and Learning. Consultado en: http://www. quasar.ualberta.ca/edpy202/edtech/mindtool.htm

Ministerio de Educación de Chile. (2002). Estudio Internacional Tecnologías de Información en el Sistema
Escolar (SITES M1). El caso de Chile. Consultado en: http://www.enlaces.cl/Despliegue_Contenidos. php?id_seccion=1\&id_contenido $=32$

Norman, Donald A. (1993). Things that make us smart. Reading, Massachusetts: Addison-Wesley.

Organisation for Economic Cooperation and Development (OECD). (2001). Learning to Change: ICT in Schools. París: OECD.

Organisation for Economic Cooperation and Development (OECD)/CERT. (1999). ICT: School Innovation and the Quality of Learning - Progress and Pitfalls. París: OECD.

Pelgrum, W. J. (2001). Obstacles to Integration of ICT in Education: results from a Worl Wide Education Assesment. Computers \& Education, 37, 163-178.

Perkins, David. (1986). Marcos de pensamiento. Material traducido de: Thinking frames. Educational Leadership, 43(8), 4-10.

Roshelle, J. M.; Pea, R. D.; Hoadley, C. M.; Gordin, D. N. y Means B. M. (2000). Changing How and What Children Learn with Computer-Based Technologies. Children and Computer Technology, 10, 76-101.

Sánchez, J. (2003). Integración curricular de TIC: concepto y modelos. Revista Enfoques Educacionales, 5(1), 51-65.

Sánchez, J. (2000). Aprender con Internet: mitos y realidades. Actas V Congreso Iberoamericano de Informática Educativa, RIBIE. Santiago de Chile: Universidad de Chile.

Sánchez, J. (1993). Informática Educativa. Santiago de Chile: Editorial Universitaria.

Tascón Trujillo, Claudio. (2004). La potenciación de aprendizajes en un entorno TIC: los mapas conceptuales como instrumento cognitivo y herramienta de aprendizaje visual. Primer Congreso Internacional sobre Mapas Conceptuales. 14-17 de septiembre. Pamplona, España. 